\title{
Dípteros acuáticos de dos humedales de la costa central de Perú y obtención de adultos a partir de pupas en el laboratorio
}

\author{
Rodolfo Mauricio Castillo-Velásquez ${ }^{\star}$, Ernesto W. Alvarado-García, \\ Adriana R. Laurent-Rios, Ana A. Huamantinco-Araujo
}

Laboratorio de Invertebrados Acuáticos, Facultad de Ciencias Biológicas, Universidad Nacional Mayor de San Marcos, Perú.

\section{Material Suplementario}

Puparios identificados en el presente estudio:

Familia Ephydridae

Subfamilia Ephydrinae

Tribu Ephydrini

Género Ephydra

Especie Ephydra gracilis

Género Neoephydra

Género Setacera

Tribu Scatellini

Género Scatella

Tribu Dagini

Género Brachydeutera
Subfamilia Hydrelliinae

Tribu Dryxini

Género Paralimna

Subfamilia Discomyzinae

Tribu Discomyzini

Género Mimapsilopa

\section{Comentarios generales}

Los tres primeros puparios (Figuras 1S, 2S y 3S), pertenecientes a la Tribu Ephydrini, presentan como características generales: (a) un tubo respiratorio elongado y bifurcado, (b) presencia de ocho segmentos con propatas ventrales (5-12), siendo la última de mayor tamaño que las demás y (c) un patrón de espinas bien marcado en la parte dorsal. Mathis y Marinoni (2016) resaltan estas características en larvas de último estadio, añadiendo la presencia de espinas en las propatas en filas bien definidas y espiráculos anteriores con 2-8 papilas marginales. En los cuatro puparios restantes (Figuras 4S, 5S, 6S y 7S) se puede observar la ausencia de propatas y un tubo respiratorio reducido o sésil. En el caso del género Brachydeutera, podemos observar los espiráculos posteriores truncados y en la parte anterior la presencia de un órgano respiratorio en forma de espátula curvada bien desarrollada, que no siempre es visible en las larvas, pues en 
muchas de ellas luego de ser fijadas con alcohol se contraen escondiendo estas estructuras. Scatella presentó en su pupario pequeñas setas que no forman manchas ni patrones dorsales definidos como en Ephydrini, mas si son uniformes en casi toda su superficie; además, poseen un espiráculo anterior con cinco papilas. Para la tribu Discomyzini, Mathis, et al. 2015, menciona que los espiráculos anteriores de las larvas tienen forma de coliflor (cauliflower), en el pupario de Mimapsilopa se observó algo similar, presentando como estructura central un espiráculo de apariencia bifurcada y con un número de papilas variable (7-8), cabe resaltar que estos espiráculos anteriores fueron montados en laminas, por lo que es posible que se hayan perdido algunos detalles de su forma. Paralimna (de la tribu Dryxini) presentó un espiráculo bifurcado bastante desarrollado y con ocho aberturas por cada extremo. Finalmente, es importante mencionar que solo a nivel de subfamilia (e.g. Ephydrinae), las larvas y puparios pueden presentar una gran variabilidad entre éstas (Fig 3S-6S), por lo que no se recomienda utilizar este trabajo como una clave, si no como un complemento en la identificación de estadios inmaduros de Ephydridae mencionados en el presente artículo.

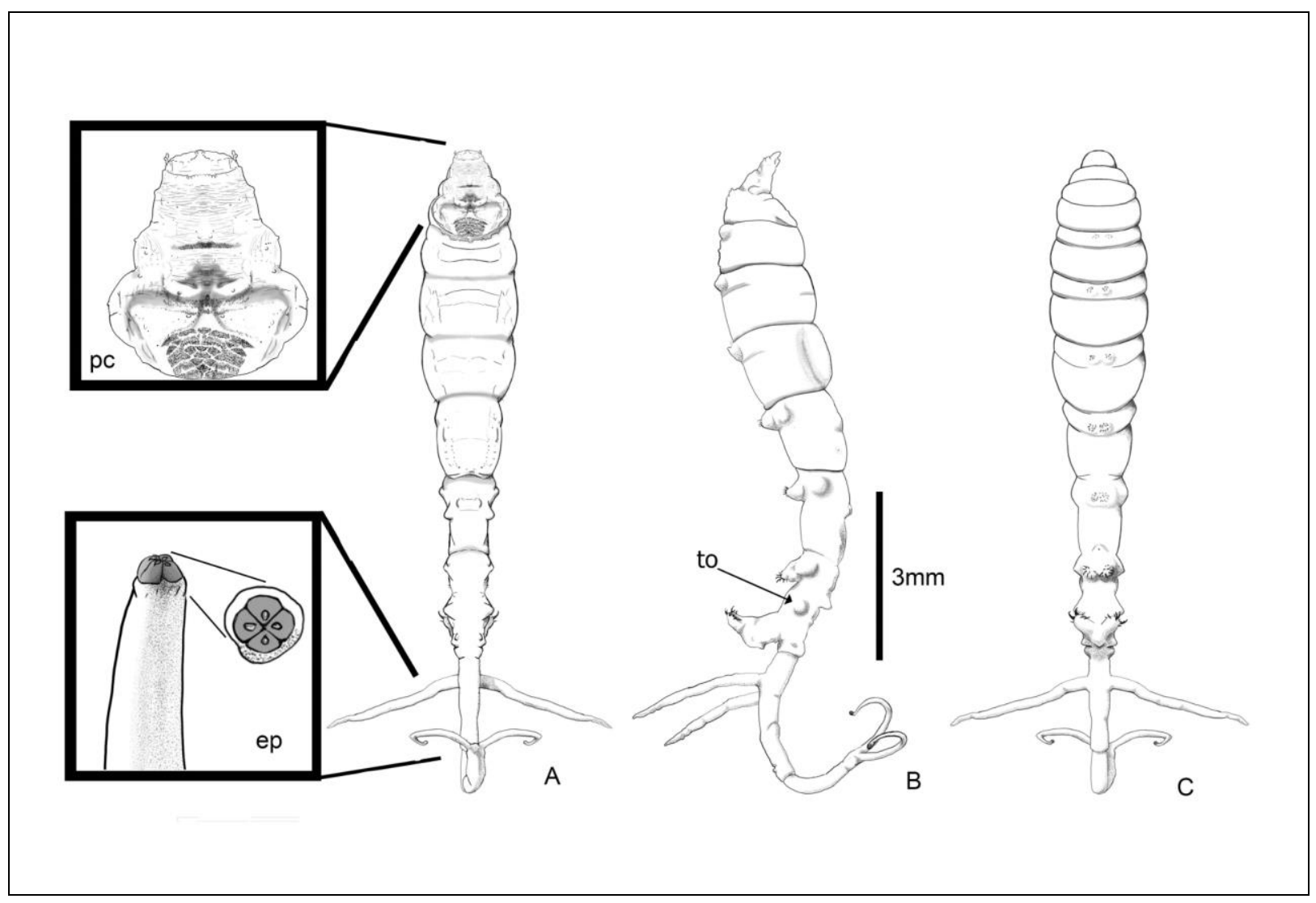

Figura 1S. Pupario de Ephydra gracilis. (A) Vista dorsal, (B) Vista lateral, (C) Vista ventral. En vista dorsal (A) podemos observar los doce segmentos del pupario, los espiráculos anteriores y la placa cefálica (pc), esta última presentó una ornamentación simétrica, con setas muy delgadas en el área central, además, cerca de la base de la placa posee un patrón semicircular formado por varios cúmulos de setas. En vista lateral (B) se pueden observar propatas en los segmentos del 5-12, siendo el último par de mayor tamaño que las demás, la cual sobresale y se curva ligeramente hacia adelante, también presenta tubérculos ovoides (to) sobre todo en los últimos segmentos de la zona posterior. En vista ventral (C) se pueden notar las espinas curvas fuertemente desarrolladas de las propatas del séptimo y octavo segmento. En todas las vistas es posible observar en la zona proximal del tubo respiratorio dos filamentos accesorios y en la zona distal una bifurcación con cuatro espiráculos posteriores (ep) en cada extremo. 


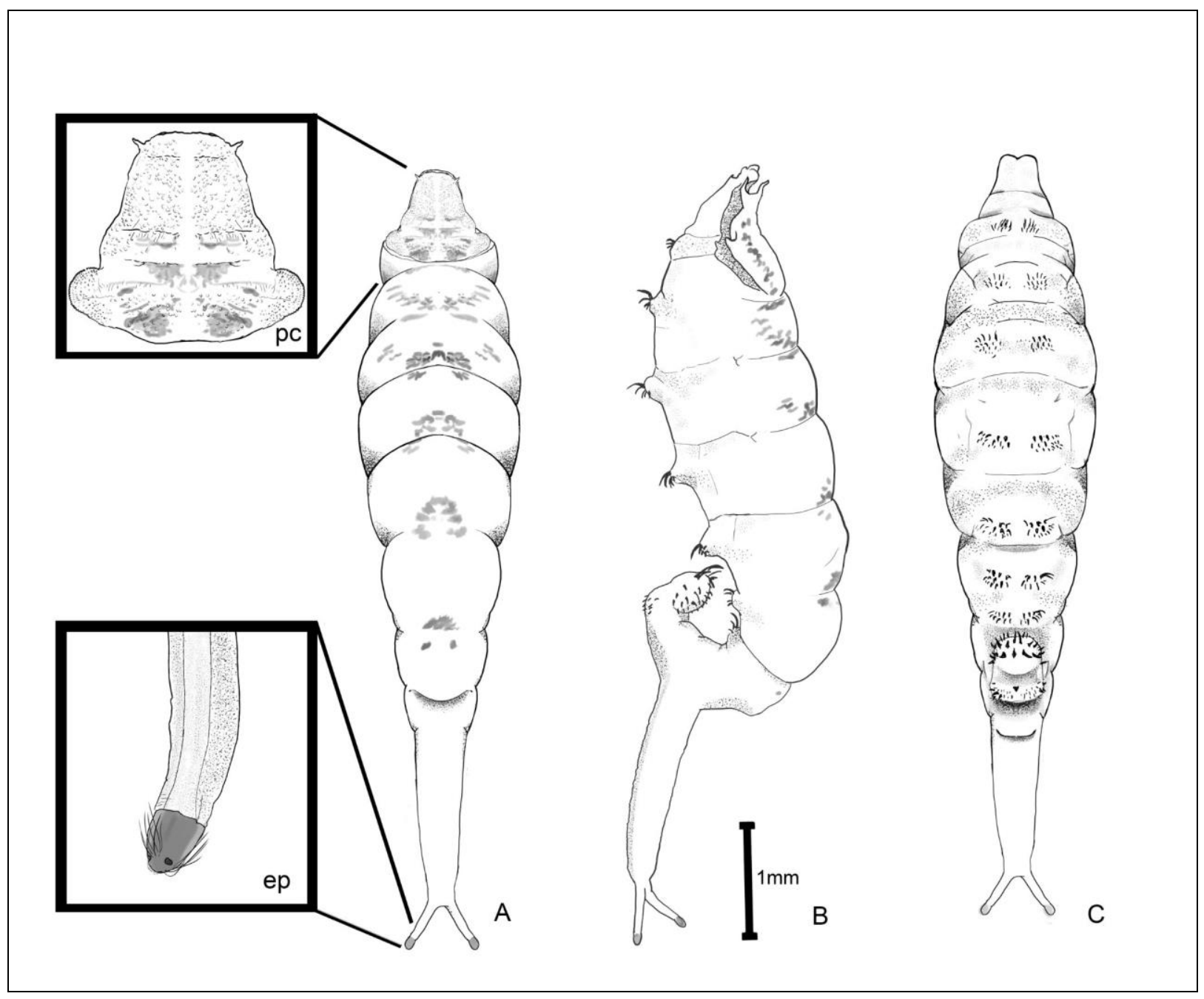

Figura 2S. Pupario de Neoephydra sp. (A) Vista dorsal, (B) Vista lateral, (C) Vista ventral.

En vista dorsal (A) se puede observar los espiráculos anteriores y la placa cefálica (pc), esta última presenta poca ornamentación en la zona central formando una pequeña banda longitudinal estrecha, en la región anterior de la placa cefálica se observa los espiráculos respiratorios anteriores, que presentan tres papilas cada uno. En vista lateral (B) presenta propatas en los segmentos del 5-12, la última propata sobresale y se curva hacia delante formando un anillo bien marcado. En vista ventral (C) se puede observar bandas de setas paralelas a las estrías que recorren el cuerpo, y pequeños cúmulos de setas en posición anterior y hacia los lados de cada propata; además, en esta vista se puede notar la placa anal elevada por la curvatura de la última propata. En la parte posterior del pupario se extiende un tubo respiratorio bifurcado que posee en cada extremo un espiráculo posterior (ep) con tres aberturas y rodeado por largas setas hidrófobas. 

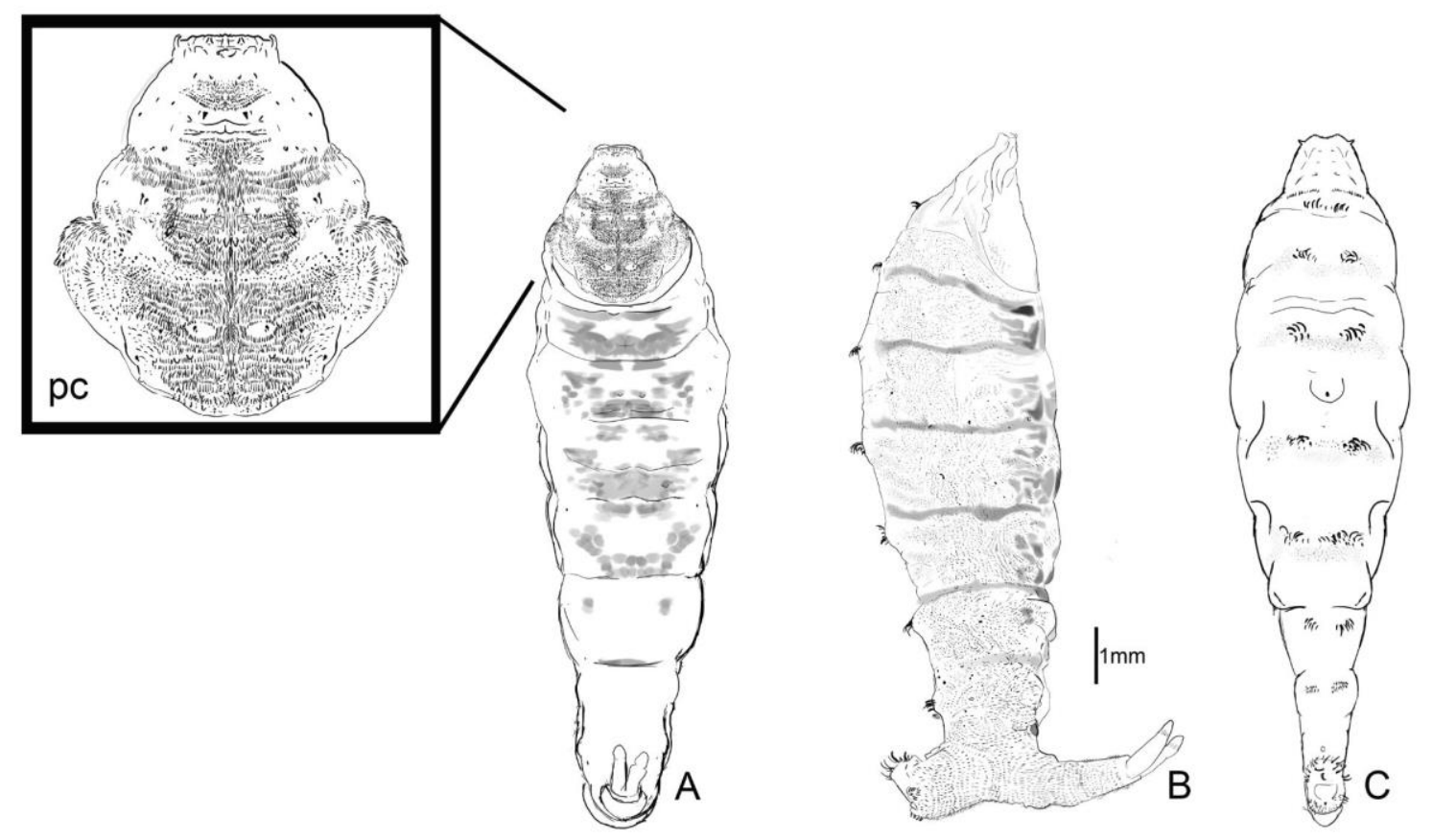

Figura 3S. Pupario de Setacera sp. (A) Vista dorsal, (B) Vista lateral, (C) Vista ventral.

En la vista dorsal $(A)$ se aprecian patrones de manchas que se repiten de forma similar en los primeros segmentos posteriores a la placa cefálica (pc). La placa cefálica (pc) se encuentra fuertemente ornamentada, incluyendo los espiráculos respiratorios anteriores y las setas acumuladas en un patrón semicircular continuo, con una estría que se curva hacia el extremo anterior; la región medial de la placa cefálica inicia con varias filas transversales y culmina con una fila longitudinal de setas cortas con aspecto de puntos; la región terminal de la placa es muy densa, con dos áreas centrales que poseen setas muy cortas y rodeando un par de setas más gruesas. En vista lateral (B) se pueden observar cinco franjas transversales oscuras en la región media del pupario, todos los segmentos que poseen propatas presentan espinas curvas y la última propata es de mayor tamaño, con espinas arqueadas hacia el extremo anterior. En vista ventral (C) nuevamente se aprecia las espinas curvas en las propatas de los segmentos del 5-12, el último par de propatas está fusionado en una sola estructura y la curvatura basal expone la placa anal. 

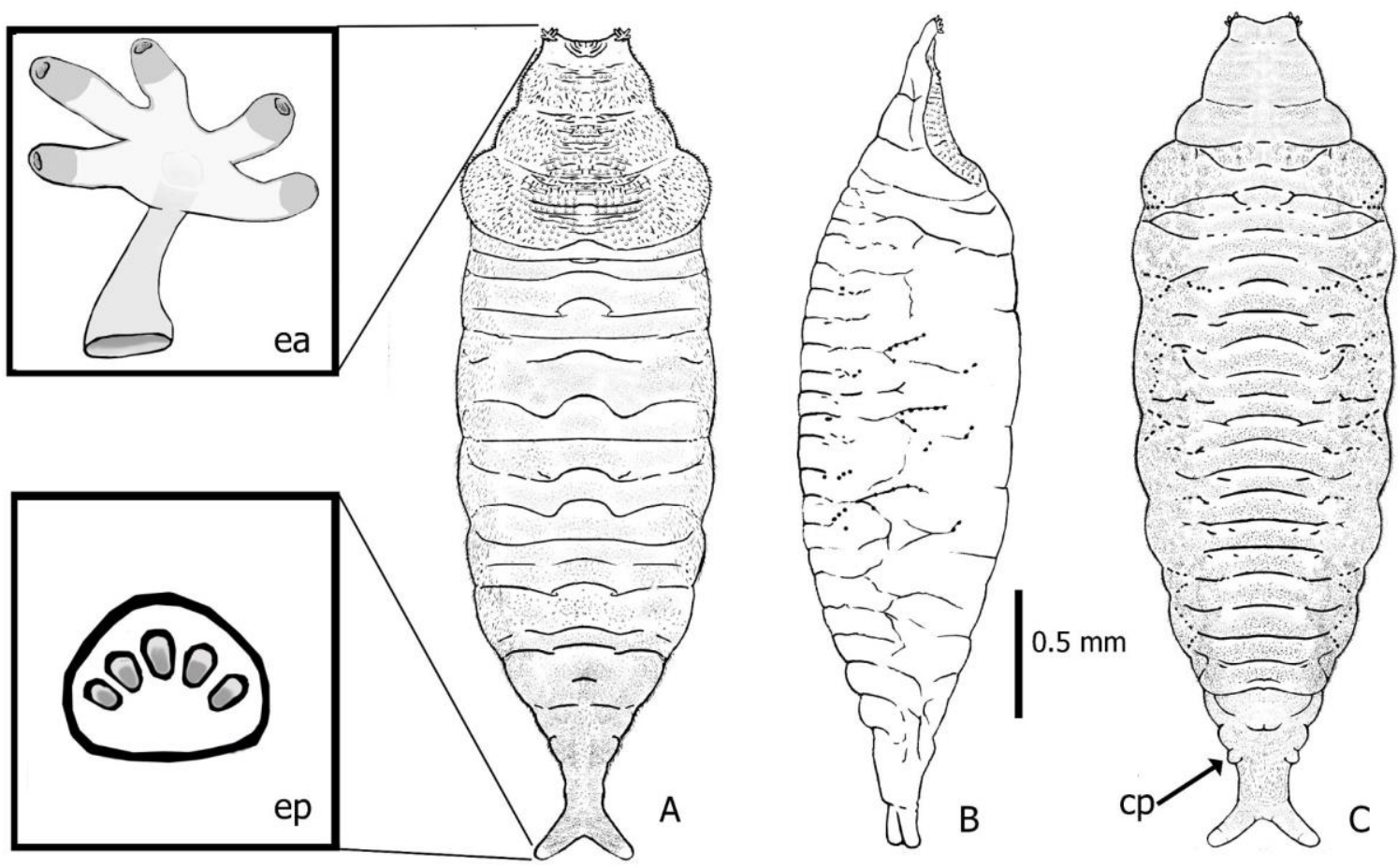

Figura 4S. Pupario de Scatella sp. (A) Vista dorsal, (B) Vista lateral, (C) Vista ventral.

En vista dorsal (A) se aprecian estrías que dividen el cuerpo con patrones similares, muchas de estas se curvan fuertemente hacia el extremo anterior al llegar al centro del pupario, también se puede ver la placa cefálica, la cual es simétrica y presenta un patrón regular de pequeñas espinas en toda su superficie, además, a cada lado del extremo anterior presenta un espiráculo respiratorio ramificado (ea) con 5 papilas. En vista lateral $(B)$ y ventral $(C)$, se observa que el pupario presenta setas de tamaño uniforme y estrías cuyos patrones no se mantienen a lo largo del pupario, así mismo en el extremo proximal del tubo respiratorio posterior presenta dos tubérculos pronunciados (cp). Este tubo respiratorio bifurcado es reducido y presenta en cada extremo un espiráculo con cinco aberturas dispuestas como en (ep). 

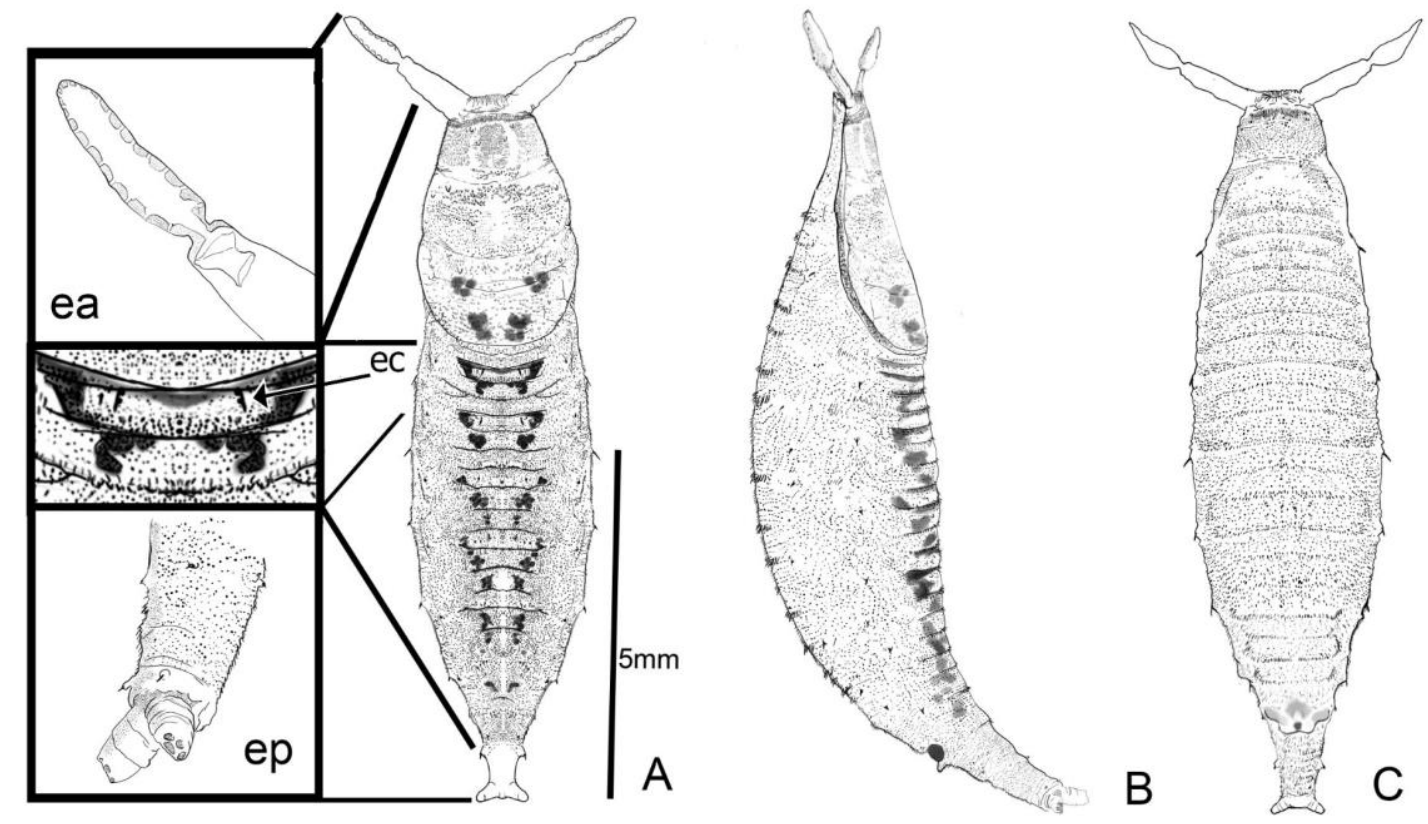

Figura 5S. Pupario de Brachydeutera sp. (A) Vista dorsal, (B) Vista lateral, (C) Vista ventral.

En vista dorsal $(A)$ muestra patrones de estrías horizontales cuya extensión va disminuyendo del extremo anterior al posterior, posee patrones simétricos de manchas como se aprecia en la figura, al inicio de cada patrón se ubica un par de tubérculos con espinas (ec) resultando en un total de hasta seis pares. En vista lateral (B) se observó dos filas irregulares de ocho tubérculos que terminan en pequeñas espinas, solo en la vista ventral y dorsal es notoria una de las filas con las espinas marginales a los lados del pupario. En la vista ventral (C) se puede apreciar un patrón repetido de aproximadamente 20 bandas de setas transversales hasta la placa anal, en el extremo posterior del pupario presenta un tubo respiratorio bifurcado (ep) con los extremos truncados, cada extremo tiene tres aberturas que rodean una "cicatriz espiracular" (spiracle scar). En la placa cefálica, la región anterior muestra una separación de los tubos respiratorios anteriores con una franja de setas y una estría transversal que llega casi hasta los bordes; la región medial posee una gran cantidad de tubérculos de tamaño variable y termina en dos estrías transversales que se encuentran en el centro; la región terminal posee gran cantidad de setas y tubérculos de tamaño variable, destacando cuatro manchas, dos manchas separadas y dos más cercanas. Cada uno de los tubos respiratorios anteriores (ea) tiene la forma de un pedúnculo con un extremo similar a una hoja espatulada curvada, la cual posee aproximadamente 22 aberturas espiraculares. 

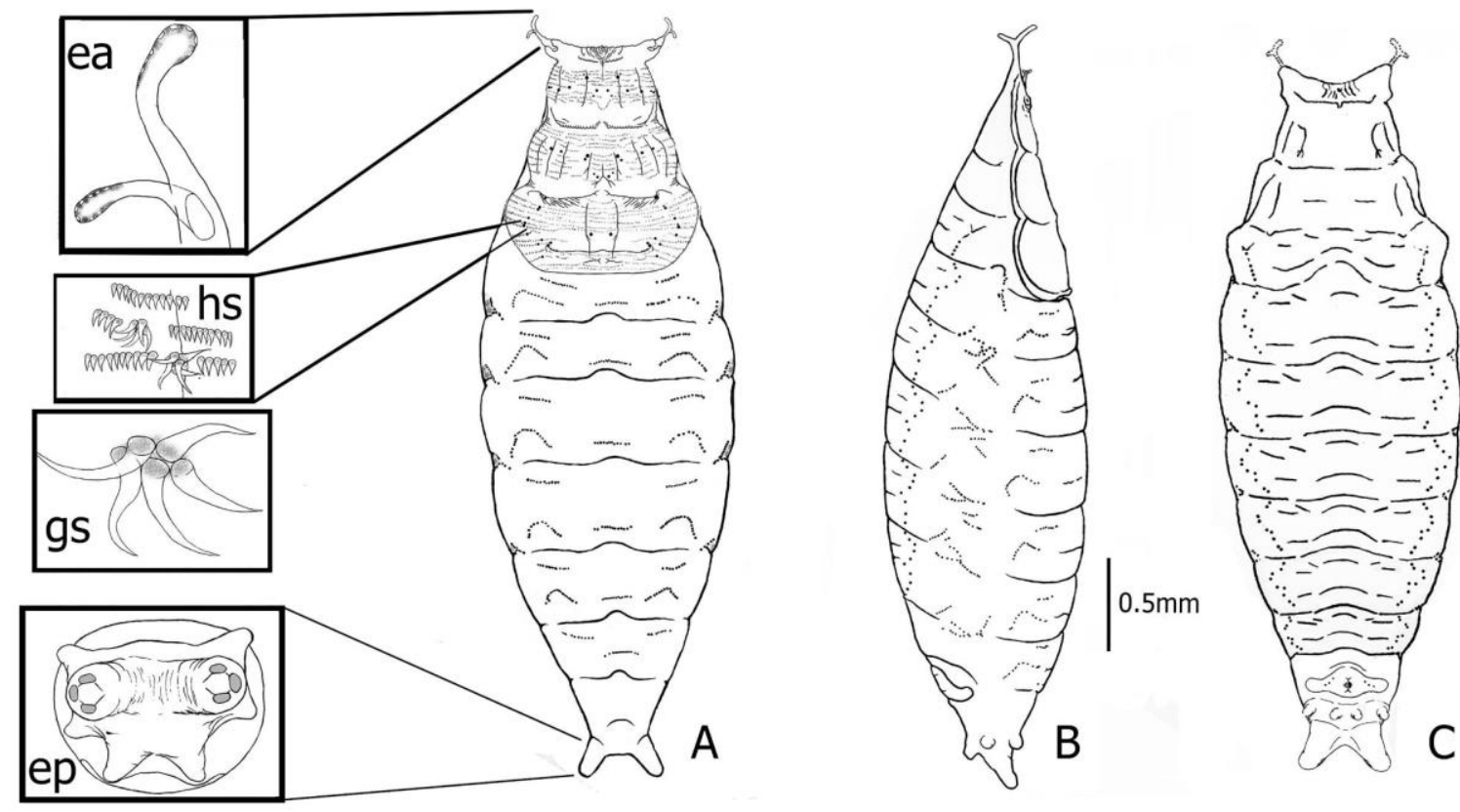

Figura 6S. Pupario de Paralimna sp. (A) Vista dorsal, (B) Vista lateral, (C) Vista ventral.

El pupario de Paralimna sp. posee una ornamentación que se mantiene en todos sus segmentos. En vista dorsal (A) se observó estrías transversales longitudinales con una pequeña curvatura en el centro y cercanas a estas, filas rectas y semicirculares de setas en los extremos. En vista lateral $(B)$ se pueden apreciar grupos de setas poco uniformes; y en la vista ventral $(C)$ se observó en cada lado del pupario una fila de setas en zig-zag en orientación longitudinal. La placa cefálica visible en vista dorsal posee dos espiráculos respiratorios bifurcados (ea) con ocho aberturas en cada extremo, la ornamentación de la placa se compone de cinco regiones separadas por interrupciones causadas por el adelgazamiento de las setas, que culminan en estrías. Las hileras de setas de la placa cefálica (hs) presentó agrupaciones variables de 5-6 setas (gs) espaciadas en patrones regulares. En la parte posterior se observó un par de espiráculos respiratorios (ep) que poseen tres aberturas cada uno; finalmente, posterior a la placa anal, presenta seis tubérculos grandes: cuatro en vista ventral y dos más en vista lateral. 

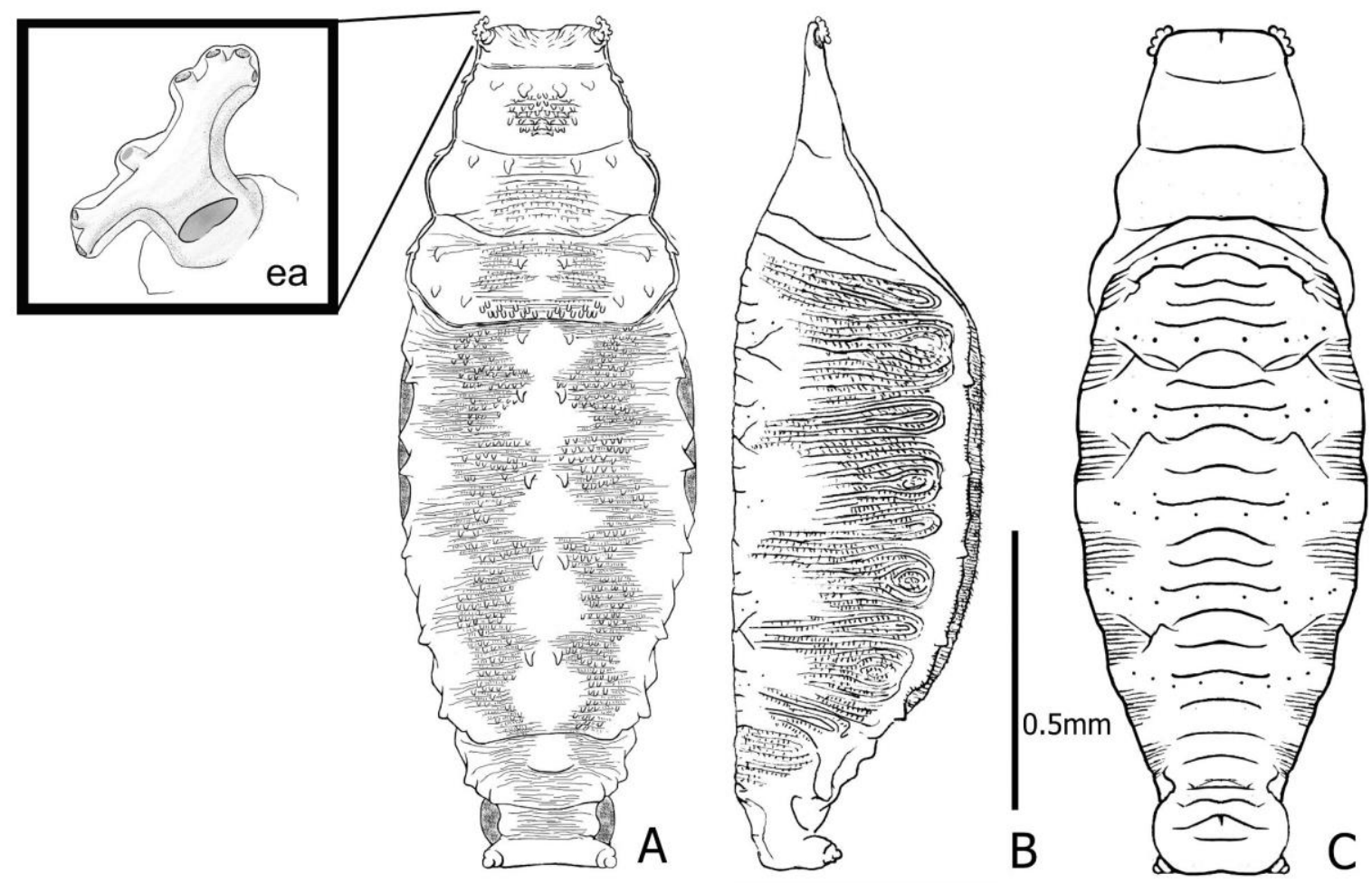

Figura 7S. Pupario de Mimapsilopa sp. (A) Vista dorsal, (B) Vista lateral, (C) Vista lateral.

El pupario de Mimapsilopa sp. se encuentra fuertemente ornamentado, la vista dorsal (A) presenta dos bandas longitudinales de estrías y tubérculos en zig-zag, dejando un patrón claro en forma de una fila de rombos en el centro del pupario, además, en esta área se observaron dos filas de tubérculos de mayor tamaño que los demás; y a cada lado del pupario presenta seis tubérculos pronunciados. La placa cefálica presenta cuatro regiones que son divididas por tres estrías transversales que llegan casi hasta el extremo de la placa, estas estrías son inmediatamente seguidas por una fila de seis tubérculos; la primera región no está muy ornamentada y en esta se encuentran los espiráculos respiratorios anteriores (ea) en la parte superior, los cuales son bifurcados y con un número de papilas variable (7-8); el segundo segmento posee un cúmulo de estrías transversales y tubérculos en el centro; la tercera región posee estrías transversales en el centro que se extienden un poco más hacia los lados, debajo de cada una de éstas hay una hilera de setas; la última región de la placa cefálica posee las estrías en zig-zag y las setas que dan inicio al patrón de rombos que continúa en el pupario, en la base de la placa se agrupan varios tubérculos pequeños y a cada lado hay dos tubérculos de mayor tamaño. En vista lateral (B) las estrías transversales tienden a curvarse hacia el lado ventral del pupario, donde llegan a agruparse y formar, entre seis y nueve, patrones circulares bien definidos. En vista ventral $(C)$ se puede apreciar seis filas de puncturas transversales y entre cada una de estas filas se observaron tres estrías paralelas centrales. 